\title{
Case Report on Secondary Complications of Neck Dissection Surgery
}

\author{
Sandeep Babasaheb Shinde ${ }^{1}$ \\ ${ }^{1}$ Department of Surgery, Krishna Institute of Medical Sciences (Deemed to Be University), Karad, Maharashtra, India.
}

\section{INTRODUCTION}

The most common malignant neoplasm which affects structures of oral cavity is squamous cell carcinoma (SCC). Common sites of oral SCC are tongue and floor of mouth followed by buccal mucosa, gingiva and soft palate. Predisposing factors such as smoking, tobacco chewing along with alcohol consumption leads to carcinoma. SCC of gingiva located in keratinized portion is normally painless. Yoon et al described the clinical aspect of oral SCC which can vary from white plaque to an infected ulcerative lesion ${ }^{1}$. Gingiva squamous cell carcinoma shows features like exophytic mass with granular, papillary or present as ulcer. Patient comes to the doctor with predominant symptom like gum pain. Premolar and molar lesion are common sites and lower jaw is likely affected more than upper jaw. ${ }^{2}$

Gingiva SCC is mostly asymptomatic in nature during early phase and frequently misdiagnosed as inflammatory lesion of oral cavity such as periodontitis, pyogenic granulomas, and inflammatory hyperplasia. Misinterpreted or delayed diagnosis and treatment leads to slow prognosis. ${ }^{3}$

The gold standard treatment for Head and Neck cancer is surgery combined with radiotherapy or chemotherapy. Surgery often leads to dissection of lymph nodes because lymph nodes are the main source of further spread of metastasis. Patient who had undergone to neck dissection surgery for head and neck cancer should start early physical therapy rehabilitation program. Along with home program therapist assisted physical therapy sessions leads to early betterment of patient and early prognosis. ${ }^{4}$ Physical therapy plays important role in post-operative period. It reduces post-surgical immobility period in order to reduce further post-surgical complication as well as minimize chances of developing malfunction of shoulder joint. Follow up for long period is necessary. Early intervention of physical therapy is helpful for reducing pain, maintaining arm mobility. 4

Immediately after surgery of head and neck cancer, reduced range of head and neck as well as reduced and painful range of shoulder becomes more prominent. Post-operative functional impairment affect the quality of life so early rehabilitation including speech therapy, physiotherapy should be intensified as early as possible. ${ }^{5}$

The most common malignant neoplasm which affects structures of oral cavity is squamous cell carcinoma. The gold standard treatment for Head and Neck cancer is surgery combined with radiotherapy or chemotherapy. Surgery often leads to dissection of lymph nodes because lymph nodes are the main source of further spread of metastasis. Patient who had undergone to neck dissection surgery for head and neck cancer should start early physical therapy rehabilitation program. Along with home program therapist assisted physical therapy sessions leads to early betterment of patient and early prognosis. Post-operative functional impairment affect the quality of life so early rehabilitation including speech therapy, physiotherapy should be intensified as early as possible. Post operatively patient tend to take rest without doing any mobility exercises due to pain which further leads to the restriction and complication of the joint.
Corresponding Author: Dr. Sandeep Babasaheb Shinde, Associate Professor, Faculty of Physiotherapy, Krishna Institute of Medical Sciences, Deemed to be University, Karad, Maharashtra, India. E-mail: drsandeepshinde24@gmail.com

DOI: $10.14260 / j e m d s / 2020 / 561$

How to Cite This Article:

Shinde SB. Case report on secondary complications of neck dissection surgery. $J$ Evolution Med Dent Sci 2020;9(35):25802583, DOI: $10.14260 /$ jemds/2020/561

Submission 31-03-2020,

Peer Review 15-07-2020,

Acceptance 22-07-2020,

Published 31-08-2020.

Copyright (c) 2020 JEMDS. This is an open access article distributed under Creative Commons Attribution License [Attribution 4.0 International (CC BY 4.0)] 


\section{PRESENTATION OF CASE}

A 72 years old male patient came to the OPD of dental school of examination with a complaint of white painless patches over lower region of tooth. The patient reported irritation of gingiva adjacent to tooth. Dentist prescribed him antibiotics, analgesics and chlorhexidine mouthwash for five days. No changes in clinical symptoms were observed. Within the next three months, patient developed yellow whitish plaques over lower aspect of the tooth. The patient thus went to periodontist where intraoral clinical examination was done and showed recession of marginal gingiva. No other periodontal involvement detected. Other mucosal membranes were within normal range. Patient was suggested to do superficial biopsy which was having no any clinical significance. So deep excisional biopsy was taken which was clinically significant which showed ulceroproliferative growth of about $2 \times 2 \mathrm{~cm}$, present over left side of gingiva buccal sulcus in the region of 31 to 33. Patient was habited to tobacco chewing for 8 to 10 years along with smoking and alcohol drinking.

\section{CLINICAL DIAGNOSIS}

Cluster of Secondary Complications of Neck Dissection Surgery.

Due to lack of awareness of post-operative physiotherapy treatment, patient ended up in secondary complications like neck stiffness, painful shoulder, deformities, and deviated mouth. If the patient would have been aware of importance of physiotherapy treatment post operatively, quality of life of patient would be better than now.

\section{PATHOLOGICAL DISCUSSION}

For the diagnostic purpose, specimen of about $1 \times 1 \mathrm{~cm}$ incised from left side interdental papilla 33 and one more specimen was excised from labial vestibule from 31 region. Macroscopic findings of bottle 1 which received single bit of soft tissue which was brownish black in color, firm in consistency with irregular surface and edges. Macroscopic examination of specimen 1 shows malignant epithelial cells proliferating into the connective tissue in forms of island showing dysplastic features like increased $\mathrm{N}: \mathrm{C}$ ratio, abnormal miosis of nuclear (pleomorphic) and individual keratin pearl formation. Bottle 2 received soft tissue grayish brown in color, soft in consistency with irregular surface and edges. Macroscopic examination of specimen shows broad rete pegs with top to bottom dysplastic feature in the epithelium. As per histopathological diagnosis specimen 1 is suggestive of welldefined squamous cell carcinoma and specimen 2 carcinoma in situ respectively.

\section{DISCUSSION OF MANAGEMENT}

In view of this diagnosis patient was referred for surgery that was performed by means of removal of lesion observing composite resection of left lower alveolus with marginal mandibulectomy along with teeth. A gray white, firm ulcer infiltrative tumor measuring $2 \times 1.1 \times 0.8 \mathrm{~cm}$ is seen which was arising from mucosa.

From neck dissection received gray yellow fibrofatty tissue with attached muscle and salivary gland. 45 lymph nodes were taken out along with cut of few fibers of sternocleidomastoid muscle of left side, some fibers of trapezius muscle were dissected along with masseter and buccinators.

Post operatively patient was admitted for 7 days in the hospital where physical therapy was given for the restricted range of shoulder joint. Exercises like passive movements, active assisted movements of shoulder joint, General mobility exercises and breathing exercises for relaxation were performed as per the patient's tolerance. Patient was asked to come on OPD basis for the rehabilitation. Though patient did not take any physical therapy sessions and only did AROM for shoulder joint which was irregular. Post-surgery six months patient came to physiotherapy department with complain of severe restriction of left shoulder joint. Physical examination determined mouth deviated to left side, difficulty in opening mouth, Depressed shoulder joint with range for flexion $42^{\circ}$, extension $30^{\circ}$, abduction $25^{\circ}$. With muscle power of grade $2 /$ 5 for the shoulder flexor, extensor, abductors, adductors and rotator muscle.

\section{DISCUSSION}

The most common neoplasm of the mouth is SCC mainly affects male older than 40 years of ages per the decreasing order the most affected site in mouth are lower lip, lateral border of tongue, retro molar region floor of the mouth and then gingiva. Involvement of mandible is more than the maxilla in the SCC of gingiva. Malignancy that develops on lips, tongue, salivary gland, floor of mouth, oropharynx, buccal surface and other intraoral location refer to subgroup of Head and neck cancers as per International Classification of Disease.

In this case the patient himself is a physician by profession. Being a health care professional, he neglected and ignored the importance of physical therapy post operatively to maintain the Range of motion of shoulder joint which lead to secondary complication. In current study post operatively, patient came to physiotherapy department after 6 months regularly for 23 sessions where treatment like AROM, PROM, Shoulder mobilization, strengthening exercises, postural correction, Jaw stretching exercises which was gradually progressed in a protocol. After regular sessions of physiotherapy showed drastic improvement in strength, range, pain and quality of life of patient

Carcinoma of gingiva is most commonly misdiagnosed as one of the many inflammatory lesions of peridontium. The most common factor of this condition is smoking, tobacco chewing along with alcohol consumption. Due to similar clinical features with other common inflammatory lesion, Gingiva SCC is diagnosed late thus invasive procedure like extraction of tooth, curettage worsen the present condition.

Study done by Milan (Italy) at European Institute of Oncology, 97 patients were registered during pre-hospital period and divided in tow group. One group for patients staying distant or away from hospital and second group was 
Physio group. Patients living nearby hospital. Physical therapy booklet of exercises given to both the groups and second group was having additional therapist assisted physiotherapy session. Early intervention of physical therapy seems to be early prognosis and effective in maintaining arm mobility and reducing pain. ${ }^{4}$
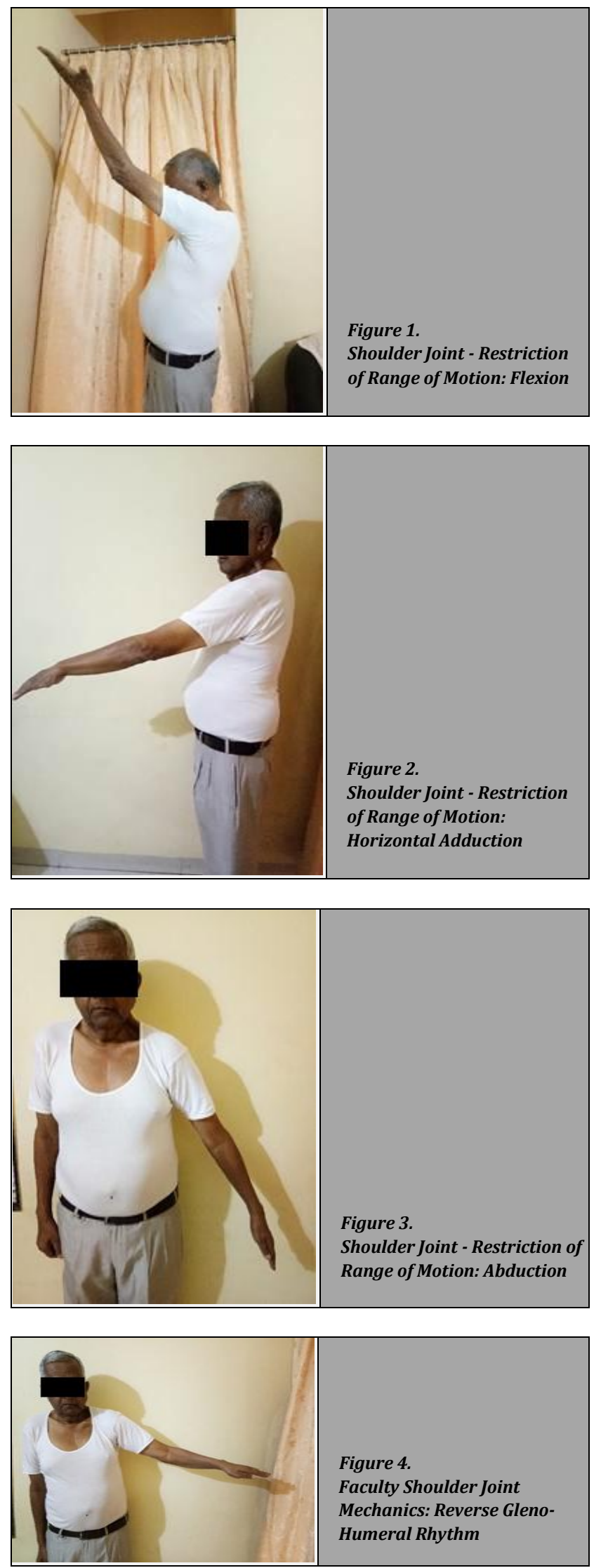

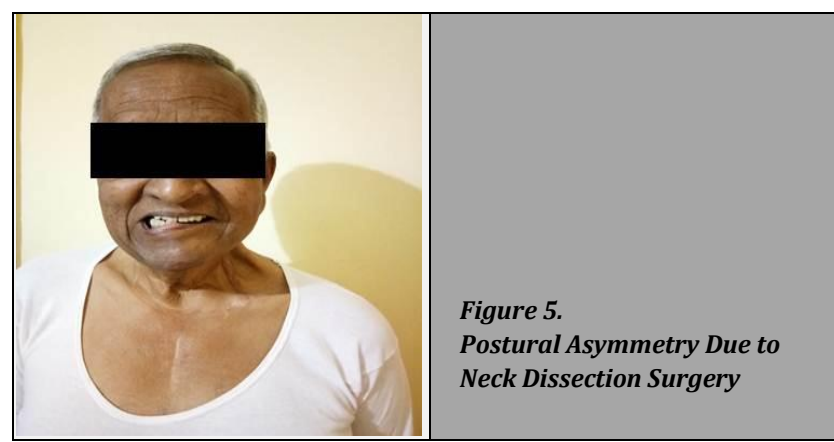

A survey conducted by Cady and Catlin $>60 \%$ gingival cancer patients are seen by dentist initially. Among 595 patients with oral cancer $52 \%$ saw a dentist as initial contact. $1 / 6^{\text {th }}$ patients were taking various therapies before they were referred for treatment of carcinoma. So, delay in the intervention of carcinoma is common in oral cancer.

Pattern and Hillel in 1993 described post-operative changes occur in shoulder joint, limited forward flexion. Reduced abduction, dull aching pain, stiffness in involved side, abnormal scapular rotation with abnormal Electromyography findings were present. ${ }^{6}$ Shah and Shinde for post-operative orthopedic conditions noted that the combination of desensitization methods along with conventional physiotherapy was effective in decreasing pain, improving ROM, and muscle strength. ${ }^{7}$ The results of this study by Sonani and Shinde supported use of closed kinetic chain exercises postoperatively are a safe and more effective for early ambulation and recovery of muscles strength. ${ }^{8}$

Salerno published a study of physical therapy protocol on pain and disability of shoulder joint after neck dissection surgeries. 15 to 30 days after surgeries intervention of physiotherapy started. After 6 months he noticed patient had undergone physiotherapy had better arm shoulder mobility than the who did not take physical therapy. Author concluded physiotherapy plays important role in for long term prevention of shoulder and neck function. ${ }^{4}$ For one of the commonest complication of neck dissection surgeries scapular position- motion programmer proved more Efficacious in reducing risk of scapular dyskinesia patients. ${ }^{9}$ The intervention of Early Physiotherapy is highly beneficial in Post-Operative Radical Mastectomy for Lymphedema. These all evidence indicates the need of early physiotherapy interventions to prevent post-operative complications of neck dissection surgery.

\section{CONCLUSIONS}

Post operatively patient tends to take rest without doing any mobility exercises due to pain which further leads to the restriction and complication of the joint. Due to lack of awareness of post-operative physiotherapy treatment patient ended up in secondary complication. If patient was aware of importance of physiotherapy treatment, post operatively, quality of life of patient would be better than now. 


\section{REFERENCES}

[1] Cabral LA, de Carvalho LF, Salgado JA, et al. Gingival squamous cell carcinoma: a case report. J Oral Maxillofac Res 2010;1(3):e6.

[2] Pyndus TA, Denga OV, Gargin VV. Complex prophylaxis of pathological changes in rats periodontal tissues with modeling of metabolic syndrome (morphological study). Вестник стоматологии 2017;4(101).

[3] Stiesch-Scholz M, Fink M, Tschernitschek H. Comorbidity of internal derangement of the temporomandibular joint and silent dysfunction of the cervical spine. J Oral Rehabil 2003;30(4):386-91.

[4] Epstein JB, Hong C, Logan RM, et al. A systematic review of orofacial pain in patients receiving cancer therapy. Supportive Care in Cancer 2010;18(8):1023-31.
[5] Horowitz AM, Nourjah P, Gift HC. U.S. adult knowledge of risk factors and signs of oral cancers: 1990. J Am Dent Assoc 1995;126(1):39-45.

[6] Borras JM, Albreht T, Audisio R, et al. Policy statement on multidisciplinary cancer care. Eur J Cancer 2014;50(3):475-80.

[7] Shah PS, Shinde SB. Effect of desensitization methods during the early mobilization phase in post-fracture conditions of upper extremity. Asian J Pharm Clin Res 2018;11(7):93-6.

[8] Jagdishbhai SR, Shinde SB. Effect of closed kinetic chain exercises in subjects with proximal femur fracture operated with dynamic hip screw and plate fixation. Indian Journal of Physiotherapy and Occupational Therapy - An International Journal 2017;11(2):98-102.

[9] Goti TS, Shinde SB. Effect of scapular position-motion maintenance exercise programme during post traumatic shoulder immobilization. Indian Journal of Public Health Research \& Development 2020;11(1):702-8. 